\title{
Calculating and visualizing the density of states for simple quantum mechanical systems
}

\author{
Declan Mulhall ${ }^{\text {a) }}$ \\ Department of Physics/Engineering, University of Scranton, Scranton, Pennsylvania 18510-4642 \\ Matthew J. Moelter ${ }^{\text {b) }}$ \\ Department of Physics, California Polytechnic State University, San Luis Obispo, California 93407
}

(Received 12 July 2012; accepted 20 February 2014)

\begin{abstract}
We present a graphical approach to understanding the degeneracy, density of states, and cumulative state number for some simple quantum systems. By taking advantage of basic computing operations, we define a straightforward procedure for determining the relationship between discrete quantum energy levels and the corresponding density of states and cumulative level number. The density of states for a particle in a rigid box of various shapes and dimensions is examined and graphed. It is seen that the dimension of the box, rather than its shape, is the most important feature. In addition, we look at the density of states for a multi-particle system of identical bosons built on the single-particle spectra of those boxes. A simple model is used to explain how the $N$-particle density of states arises from the single particle system it is based on. (C) 2014 American Association of Physics Teachers.

[http://dx.doi.org/10.1119/1.4867489]
\end{abstract}

\section{INTRODUCTION}

The concept of the density of states (DOS) is used in many areas of physics. For example, it is important for reaction rates in nuclear physics, the calculation of specific heat capacities, black-body radiation, phonon spectra, and so on. ${ }^{1-6}$ The DOS arises naturally and early in statistical physics. To calculate average quantities in statistical physics, one could do an integral over phase space, but this is typically very complex. The alternative is to express the variable of interest in terms of the energy of the system. The volume element in phase space is replaced by a weighting factor in an energy integral, which is often much easier to work with. This weighting factor is the density of states and is the subject of this paper.

Consider the problem of calculating $\langle f\rangle$, the expected outcome of a measurement of some physical quantity $f$. If you know the allowed quantum states of the system and can calculate $f_{i}$, the value of $f$ in the $i$ th state, then $\langle f\rangle=\sum_{i} f_{i} P_{i}$, where $P_{i}$ is the probability of the system being in the $i$ th state. This is sometimes referred to as a sum over microstates. It is often easier to write $f$ as a function of energy and perform a sum over the allowed energies. We then have $\langle f\rangle=\sum_{i} f\left(\epsilon_{i}\right) P\left(\epsilon_{i}\right)$, where the probability of the system being on the energy level $\epsilon$ is given by $P(\epsilon)=d_{\epsilon} e^{-\beta \epsilon} / Z$. Here $Z=\sum_{j} e^{-\beta \epsilon_{j}}$ is the partition function, $\beta=1 / k T$, and $d_{\epsilon}$ is the degeneracy of the level or number of states with energy $\epsilon$. If the system has an energy $\epsilon$, then each of these $d_{\epsilon}$ states is equally likely. Now, for our expected value of $f$ we have

$$
\sum_{i} f_{i} P_{i} \rightarrow \sum_{\epsilon} d_{\epsilon} f(\epsilon) P(\epsilon) .
$$

In a system where $\epsilon$ is continuous (or effectively so), the sum becomes an integral and $d_{\epsilon}$ is replaced by $g(\epsilon)$ - the DOS - a measure of how many states there are in a small range $d \epsilon$ around $\epsilon$. The DOS $g(\epsilon)$ is no longer an absolute number of states; it is now a weighting factor in an integral over energy. We must be careful here because $g(\epsilon)$ is often confused with the level density; indeed, the terms are often used interchangeably. We use the term "level" to mean an allowed value of energy and the DOS is the level density multiplied by a degeneracy factor.

In this paper, we present a procedural approach to obtain the DOS for systems that arise in modern physics and statistical mechanics courses. In Sec. II, we apply the procedure to a single particle in a rigid box. In Sec. III, the procedure for visualizing the DOS is summarized and the effect of the shape of the box on the DOS is examined. Section IV addresses systems of $N$ noninteracting bosons, where $N$-particle spectra are calculated and the dependence of their DOS on $N$ is compared with a simple model.

\section{VISUALIZING THE DENSITY OF STATES FOR A PARTICLE IN A BOX}

The particle in a box is one of the first examples students encounter in quantum mechanics. It is simple enough to solve from scratch by hand and exhibits much of the salient nonclassical behavior. Furthermore, it serves as a basic template for a host of interesting topics: scattering, double-well potentials, and perturbation theory to name a few. We will discuss the DOS in the context of a particle in a box of various dimensions. First, we introduce the concept of degeneracy via numerical results for the spectrum. This leads naturally to the idea of a cumulative state number from which the DOS naturally follows. Then an analytic approach to obtaining the DOS is presented.

We start with a (nonrelativistic) particle of mass $M$ inside a box. We assume the box is rigid, by which we mean that the potential energy is infinite outside the box and zero inside the box. In a one-dimensional box of length $L$ the energy levels are given by ${ }^{7} \epsilon_{n}=\epsilon_{0} n^{2}$, where $\epsilon_{0}=\pi^{2} \hbar^{2} /$ $\left(2 M L^{2}\right)$ and the quantum number $n$ is an integer. In a twodimensional square box with sides of length $L$, the energy levels are given by $\epsilon_{n}=\epsilon_{0}\left(n_{x}^{2}+n_{y}^{2}\right)$, where $n_{x}$ and $n_{y}$ are the quantum numbers corresponding to the two spatial dimensions. In the three-dimensional case of a cubical box of side length $L$, we have $\epsilon_{n}=\epsilon_{0}\left(n_{x}^{2}+n_{y}^{2}+n_{z}^{2}\right)$. 
It is natural to associate each quantum number with a number line and each integer value with a point along this line. In two dimensions the pair of quantum numbers defines a plane or two-dimensional space called $n$-space. ${ }^{8}$ This space could also be called momentum space by using the identity $p_{x}=\hbar k_{x}=n_{x} \pi \hbar / L$. The energy depends on the sum of the squares of $n_{x}$ and $n_{y}$. One way of thinking about the distribution of these energies is to locate them in $n$-space. Using the horizontal axis for $n_{x}$ and the vertical axis for $n_{y}$, we can write all energy values at their corresponding grid points. This is done in Fig. 1, where at each pair of quantum numbers $\left(n_{x}, n_{y}\right)$ the energy is written (in units of $\left.\epsilon_{0}\right)$; for example, at grid points $\left(n_{x}, n_{y}\right)=(1,3)$ and $(3,1)$ we see a "10." Suppose $n$ is the radius of a circle in $n$-space such that $n^{2}=n_{x}^{2}+n_{y}^{2}$. Rewriting the energy as $\epsilon_{n}=\epsilon_{0} n^{2}$ we see that in $n$-space a given energy (in units of $\epsilon_{0}$ ) corresponds to a circle of radius $\sqrt{\epsilon / \epsilon_{0}}$. (The radius $n$ is related to the magnitude of the momentum vector by $|\vec{p}|=n \pi \hbar / L)$. In Fig. 1, the circles corresponding to energies " 36 " and " 65 " are shown as dotted curves with radii $\sqrt{36}=6$ and $\sqrt{65} \approx 8$, respectively.

When energies correspond to more than one independent state we say they are "degenerate." If we make a list of all energies corresponding to the various quantum numbers $\left(n_{x}\right.$, $n_{y}$ ) and order them by energy, we can make a plot of the "number of states with energy $\epsilon$ " vs $\epsilon$. This quantity is $d_{\epsilon}$, the degeneracy of the energy $\epsilon$, and for discrete spectra it is an integer. A plot of $d_{\epsilon}$ is a series of spikes, of height $d_{\epsilon}$, at each allowed $\epsilon$.

To further illustrate this idea, imagine a hypothetical single-particle spectrum where the lowest nine energies are $\{\epsilon\}=\{2,2,3,3,3,3,5,5,5\}$. We have plotted $d_{\epsilon}$ vs $\epsilon$ for this system in Fig. 2 (upper left panel). At this point, it is helpful to introduce the cumulative state number $\mathcal{N}(\epsilon)$, defined as the number of states with energy less than or equal to $\epsilon$; its graph is a staircase where each step has a height $d_{\epsilon}$ and a width determined by the gap to the next energy (lower

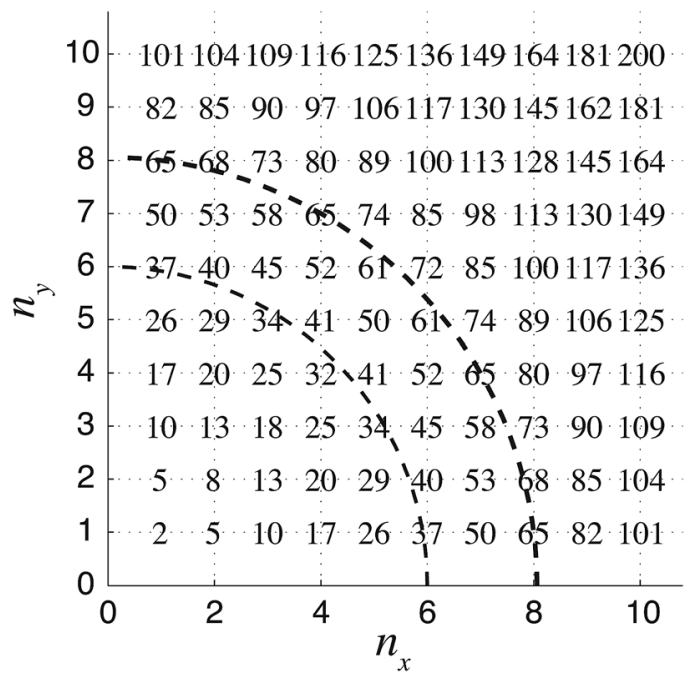

Fig. 1. The energies for different combinations of $n_{x}$ and $n_{y}$ for a particle in a 2-D square box. The numbers at the grid sites are $n_{x}^{2}+n_{y}^{2}$. The quarter circles represent constant $n=\sqrt{n_{x}^{2}+n_{y}^{2}}$; here we show $n=\sqrt{36}$ and $n=\sqrt{65}$. Certain values of $n$ give circles that intersect with the grid points. These correspond to the allowed energies $\epsilon=n^{2} \epsilon_{0}$. Notice that $n=6$ does not intersect any grid point, while $n=\sqrt{65} \approx 8$ intersects four grid points, corresponding to the degeneracy of that energy level.
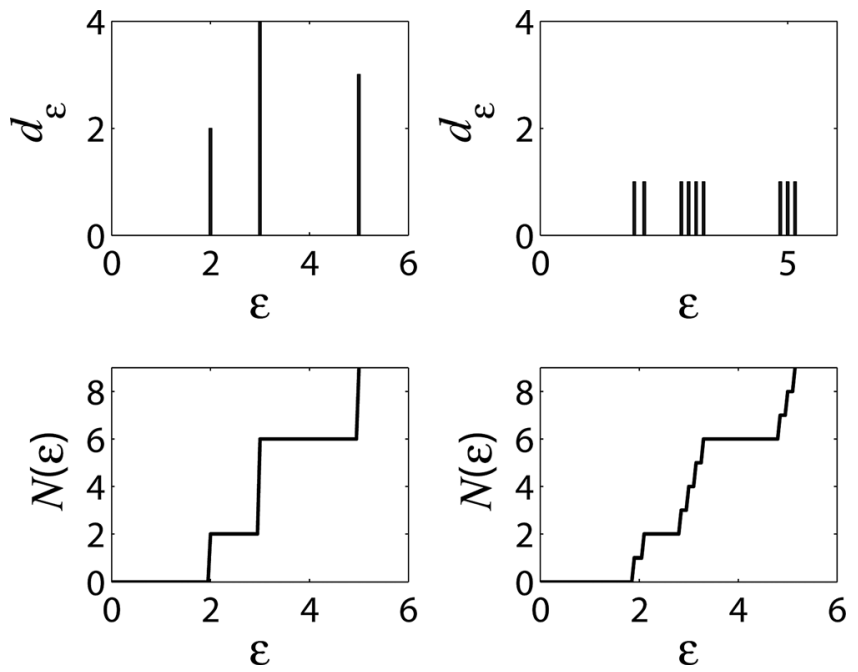

Fig. 2. The degeneracy $d_{\epsilon}$ (upper panels) and corresponding cumulative state number $\mathcal{N}(\epsilon)$ (lower panels) for the lowest nine levels of a simple, discrete system. On the left the degeneracy of the system is intact while on the right the degeneracy has been lifted. The effect on $d_{\epsilon}$ is dramatic; less so for $\mathcal{N}(\epsilon)$.

left panel of Fig. 2). In other words, given an ordered list of energies $\left\{\epsilon_{i}\right\}$ we have $\mathcal{N}(\epsilon)=i$ for $\epsilon_{i}<\epsilon<\epsilon_{i+1}$.

A plot of the spikes $d_{\epsilon}$ gives a visual measure of the degeneracies of the energies. These degeneracies are quite delicate in the sense that most perturbations to the potential will break them and the picture for $d_{\epsilon}$ will change dramatically. Each $d_{\epsilon}$-high spike will turn into a cluster of $d_{\epsilon}$ separate spikes, each one unit high. The spacing of the spikes will be determined by the strength of the perturbation. The corresponding change in $\mathcal{N}(\epsilon)$ is that each step in the unperturbed system that was $d_{\epsilon}$ high will now become a series of short steps, each one unit high (upper and lower right panels of Fig. 2). It is hoped that Figs. 1 and 2 will be a useful starting point for student discussion.

A smooth DOS function $g(\epsilon)$ is useful because at higher energies one is interested in the number of states in an interval, or the density of spikes along the $\epsilon$-axis. The DOS and cumulative state number are related by $g(\epsilon) d \epsilon=d \mathcal{N}(\epsilon)$, so the DOS is recognized as the slope of $\mathcal{N}(\epsilon)$. We must be careful here because the slope of a staircase is infinite at each step, so we mean "slope" in an averaging sense.

To get $g(\epsilon)$ from $\mathcal{N}(\epsilon)$, we can take a numerical derivative using a finite difference scheme:

$$
g(\epsilon)=\frac{d \mathcal{N}(\epsilon)}{d \epsilon}=\frac{\mathcal{N}(\epsilon+\delta \epsilon / 2)-\mathcal{N}(\epsilon-\delta \epsilon / 2)}{\delta \epsilon} .
$$

It is worth saying explicitly that if the energies are put into bins of width $\delta \epsilon$, with center $\epsilon$, then $g(\epsilon)=$ (number of states in bin)/(width of bin).

Now that we have a numerical representation for $g(\epsilon)$, we would like to get an analytic expression for $g(\epsilon)$ that is valid for the statistical region where the DOS is large and well approximated by a smooth function. We will do this for the rigid box potentials where $\epsilon=n^{2}$ (in units of $\epsilon_{0}$ ). In $n$-space, the states with energy in an interval $d \epsilon$ centered on $\epsilon$ correspond to a set of points in a spherical shell of thickness $d n$ with all-positive coordinates. In Fig. 1, the number of states between the quarter circles of radius $n$ and $n+d n$ is proportional to the area of the curved band. This result is an 
approximation, because $n_{x}$ and $n_{y}$ are discrete and there are fluctuations, but we will see that this is a good approximation nevertheless. The number of states is the "volume" of this shell but by definition it is also $g(\epsilon) d \epsilon$, so in 2-D we have $g(\epsilon) d \epsilon=(1 / 2) \pi n d n$. If $n(\epsilon)$ is a smooth function we can write $g(\epsilon)=(1 / 2) \pi n(\epsilon) d n / d \epsilon$, and using $n(\epsilon)=\sqrt{\epsilon}$ and $d n / d \epsilon=1 /(2 \sqrt{\epsilon})$ we see that the DOS is constant: $g(\epsilon)=\pi / 4$. Since the DOS is the slope of $\mathcal{N}(\epsilon)$, the function $\mathcal{N}(\epsilon)$ should be well approximated by the straight line $\mathcal{N}(\epsilon)=(\pi / 4) \epsilon$, as shown in Fig. 3. The numerical results are convincing, particularly where the energy range increases and the fluctuations about the smooth functions get smaller (see Fig. 4).

For a particle in a 3-D rigid box with side lengths $L_{x}, L_{y}$, and $L_{z}$, we will be working with a 3-D $n$-space and again each point $\left(n_{x}, n_{y}, n_{z}\right)$ corresponds to an allowed value of energy where $\epsilon=\left(\pi^{2} \hbar^{2}\right)\left[\left(n_{x} / L_{x}\right)^{2}+\left(n_{y} / L_{y}\right)^{2}+\left(n_{z} / L_{z}\right)^{2}\right] /$ $(2 M)$. For a cube of side length $L$ we have $\epsilon=\epsilon_{0} n^{2}$ with $\epsilon_{0}$ $=\left(\pi^{2} \hbar^{2}\right) /\left(2 M L^{2}\right)$ and $n^{2}=\left(n_{x}^{2}+n_{y}^{2}+n_{z}^{2}\right)$. Now the appropriate construction to get the DOS is a shell of thickness $d n$ and radius $n$ in the positive octant of a sphere, which leads to $g(\epsilon)=(1 / 2) \pi n^{2} d n / d \epsilon$. Again, using $n(\epsilon)=\sqrt{\epsilon}$ and $d n / d \epsilon$ $=1 /(2 \sqrt{\epsilon})$, we see that the DOS now has the form $g(\epsilon)$ $=(\pi / 4) \sqrt{\epsilon}$ and $\mathcal{N}(\epsilon)=(\pi / 6) \epsilon^{3 / 2}$. In Figs. 5 and 6, we compare the numerical results with these smooth functions for
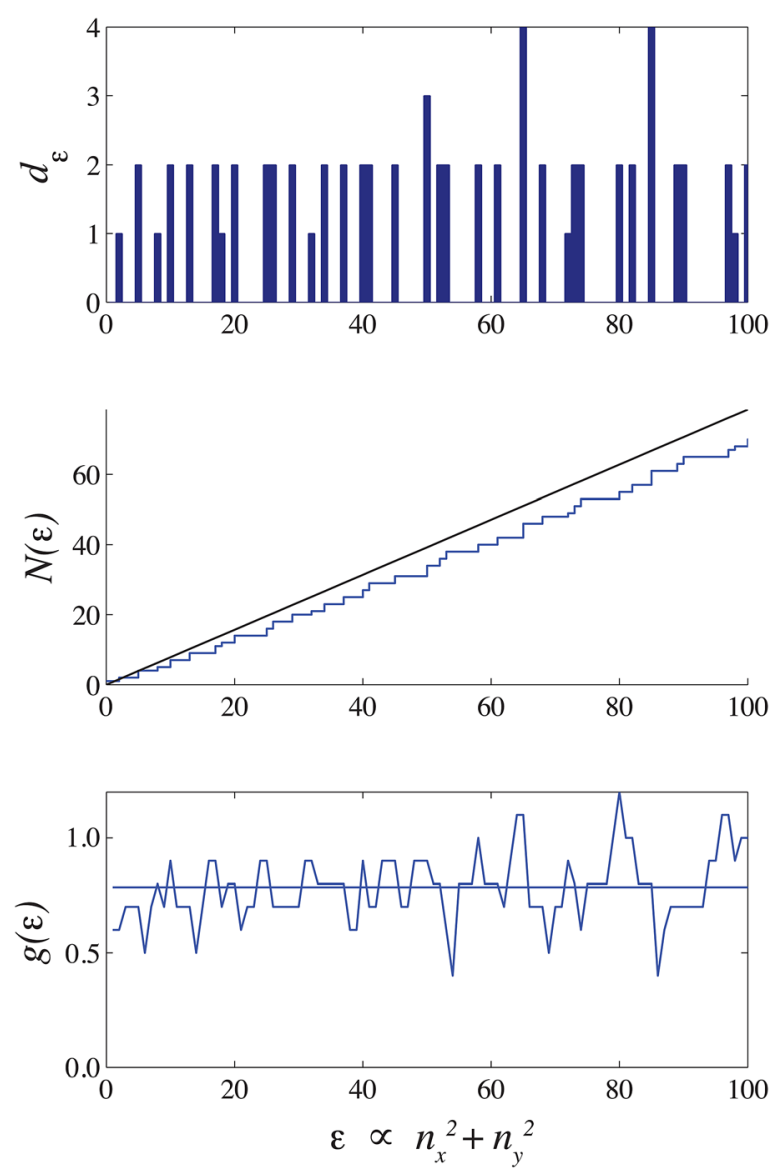

Fig. 3. Top: Degeneracy $d_{\epsilon}$ of the allowed energies $\epsilon$ (in units of $\epsilon_{0}$ ) of a single particle in a 2-D square box, up to $n_{x}=n_{y}=10$. Middle: The corresponding $\mathcal{N}(\epsilon)$. Theory (smooth line) gives $\mathcal{N}(\epsilon)=(\pi / 4) \epsilon$. Bottom: A plot of $g(\epsilon)$ obtained using the derivative scheme in Eq. (2) with a window width of 10 . Theory (smooth line) gives $g(\epsilon)=\pi / 4$.
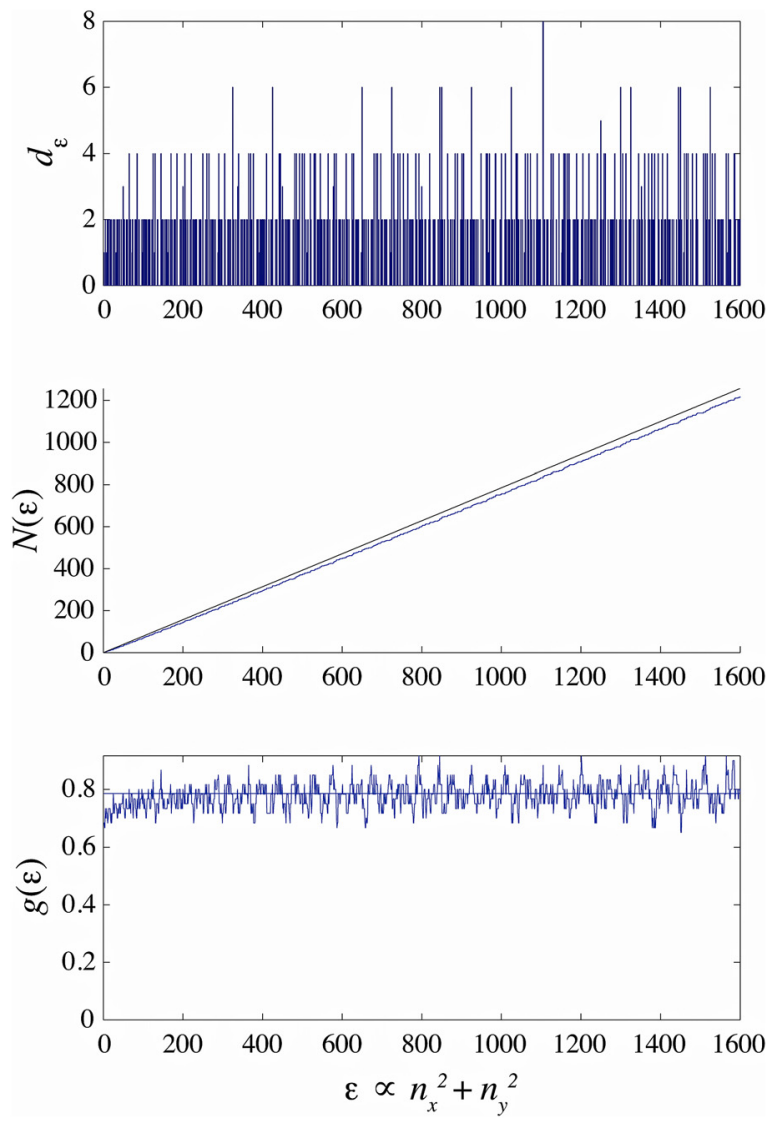

Fig. 4. Top: Degeneracy $d_{\epsilon}$ of the allowed energies $\epsilon$ (in units of $\epsilon_{0}$ ) of a single particle in a 2-D square box, up to $n_{x}=n_{y}=40$. Middle: The corresponding $\mathcal{N}(\epsilon)$. Theory (smooth line) gives $\mathcal{N}(\epsilon)=(\pi / 4) \epsilon$. Bottom: A plot of $g(\epsilon)$ obtained using the derivative scheme in Eq. (2) with a window width of 60 . Theory (smooth line) gives $g(\epsilon)=\pi / 4$.

different energy ranges. Again as the energy increases, the numerical fluctuations get smaller.

We remark here that if we have $N$ non-interacting independent particles in a rigid cube, then the total energy would be the sum of the individual energies, and this sum could be related to the surface of a $D=3 N$-dimensional hypersphere. This problem is similar to the case of a single particle in a rigid box in a $D$-dimensional space. We would need to discuss the volume of a sphere of radius $R$ in $D$-dimensions, denoted by $V_{D}$ and given by ${ }^{9}$

$$
V_{D}=\frac{\pi^{D / 2}}{\Gamma(D / 2+1)} R^{D}=C_{D} R^{D}
$$

Here, $\Gamma(D+1)=D$ !, giving $C_{2}=\pi$ and $C_{3}=4 \pi / 3$ as expected. The surface area of this $D$-sphere is given by $\mathrm{S}_{D}=D C_{D} R^{D-1}$. Where before we were concerned with the length of a quarter circle of radius $n(D=2)$ and the area of an octant of a sphere of radius $n(D=3)$, we now have the area of the positive portion of the $D$-dimensional sphere, given by $\left(1 / 2^{D}\right) S_{D}$ (the need for $n_{x}, n_{y} \ldots$ to be positive gives a factor of $1 / 2$ for each dimension). The cumulative state number is given by the volume of phase space enclosed by the boundary $n=\sqrt{\epsilon}$ :

$$
\mathcal{N}(\epsilon)=\frac{1}{2^{D}} \frac{\pi^{D / 2}}{\Gamma(D / 2+1)} \epsilon^{D / 2},
$$



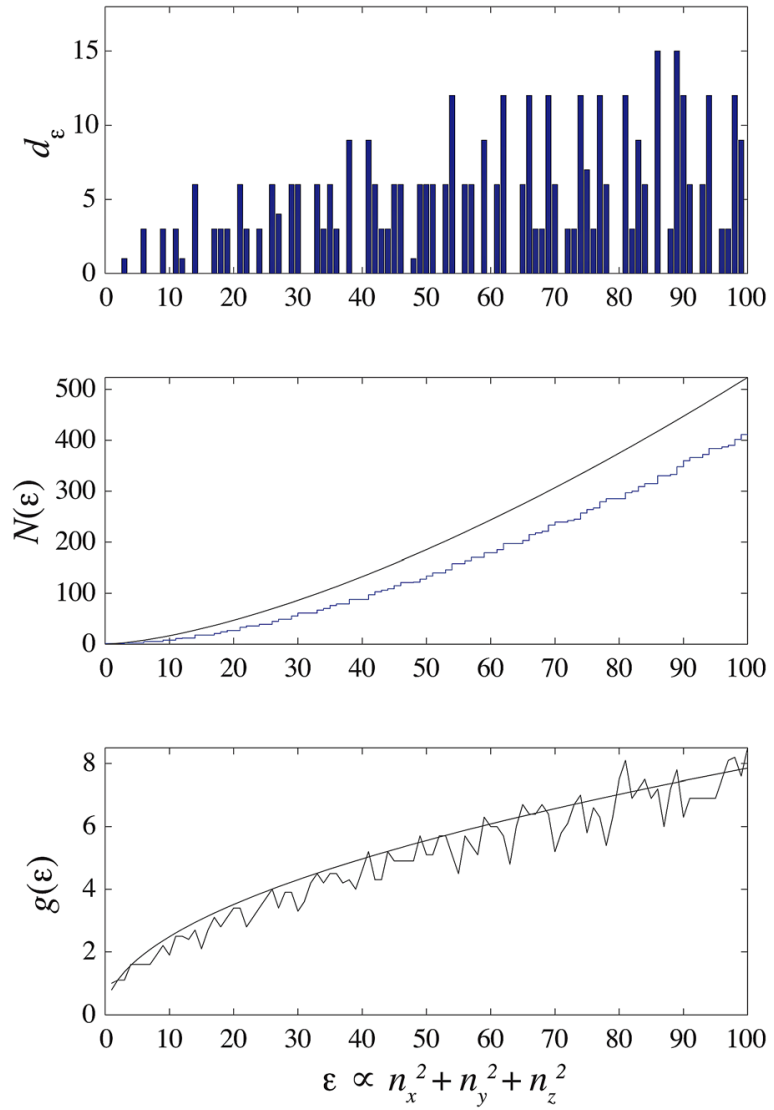

Fig. 5. Top: Degeneracy $d_{\epsilon}$ of the allowed energies $\epsilon$ (in units of $\epsilon_{0}$ ) of a single particle in a 3-D rigid cubic box, up to $n_{x}=n_{y}=n_{z}=10$. Middle: The corresponding $\mathcal{N}(\epsilon)$ is compared to the theoretical result (smooth line) $\mathcal{N}(\epsilon)=(\pi / 6) \epsilon^{3 / 2}$. Bottom: A plot of $g(\epsilon)$ obtained using the derivative scheme in Eq. (2) with a window width of 10 . Theory (smooth line) gives $g(\epsilon)=(\pi / 4) \epsilon^{1 / 2}$.

and differentiation gives

$$
g(\epsilon)=\frac{1}{2^{D+1}} D \frac{\pi^{D / 2}}{\Gamma(D / 2+1)} \epsilon^{(D / 2)-1} .
$$

The forms of $\mathcal{N}(\epsilon)$ and $g(\epsilon)$ for some representative $D$ are given in Table I. Later we will see that the DOS for one particle in $D$ dimensions is proportional to the DOS of $D$ particles in one dimension, so long as the particles do not interact.

\section{PROCEDURE FOR CALCULATING AND VISUALIZING THE DENSITY OF STATES}

For any quantum-mechanical system we can determine the cumulative state number and the corresponding density of states using the following procedure:

1. Solve the relevant quantum-mechanical problem to get a list of the energies of the system. (This can be an analytical expression or a list of energies obtained numerically.)

2. Sort the list of energies to get a set $\left\{\epsilon_{1}, \epsilon_{2}, \epsilon_{3}, \ldots, \epsilon_{n}\right\}$ up to some maximum energy $\epsilon_{\max }$.

3. Create the cumulative state number $\mathcal{N}(\epsilon)$ by making the set $\left\{\epsilon_{1}, 1\right\},\left\{\epsilon_{2}, 2\right\},\left\{\epsilon_{3}, 3\right\}, \ldots\left\{\epsilon_{n}, n\right\}$.

4. Find the DOS by taking the numerical derivative of the cumulative state number, as in Eq. (2). Choose a window size and locate the window so that its center is at $\epsilon$. The
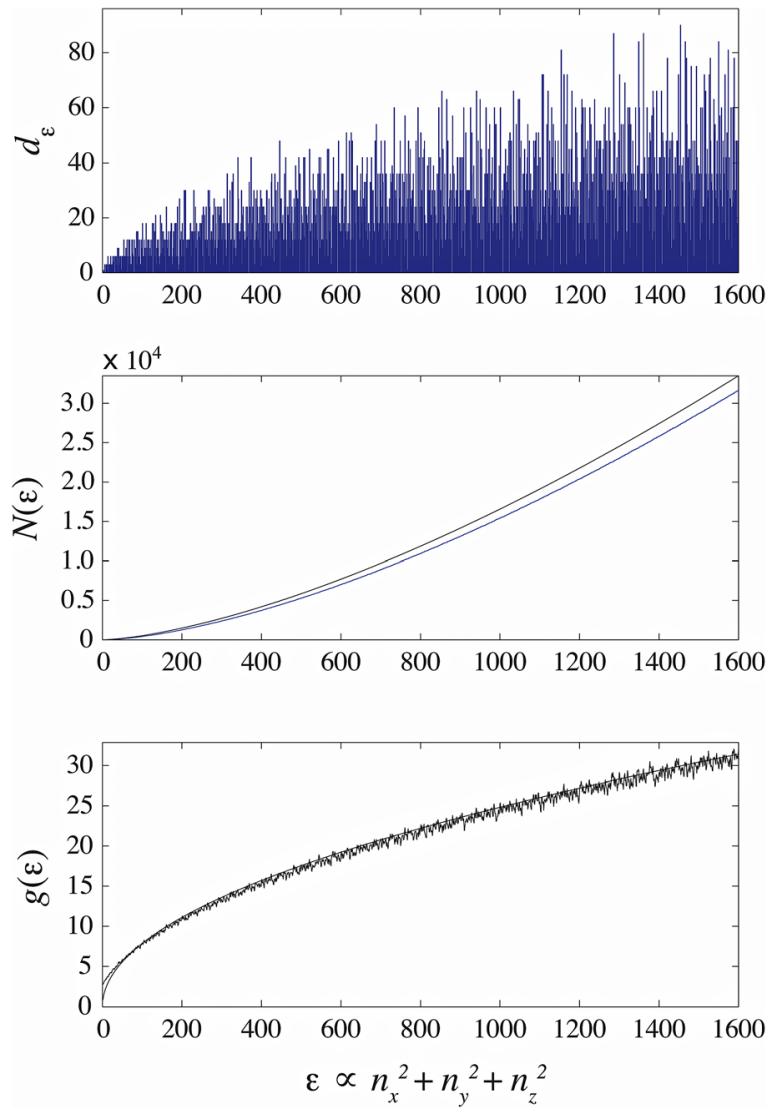

Fig. 6. Top: Degeneracy $d_{\epsilon}$ of the allowed energies $\epsilon$ (in units of $\epsilon_{0}$ ) of a single particle in a 3-D cubical box, up to $n_{x}=n_{y}=n_{z}=40$. Middle: The corresponding $\mathcal{N}(\epsilon)$ (lower curve) is compared to the theoretical result (smooth line, upper) $\mathcal{N}(\epsilon)=(\pi / 6) \epsilon^{3 / 2}$. Bottom: A plot of $g(\epsilon)$ obtained using the derivative scheme in Eq. (2) with a window width of 50. Theory (smooth line) gives $g(\epsilon)=(\pi / 4) \epsilon^{1 / 2}$.

value of $g(\epsilon)$ is the number of energies that are within that window divided by the window size. Evaluate this for all locations of the window, and you now have a list of ordered pairs $\{\epsilon, g(\epsilon)\}$.

As supplementary materials ${ }^{10}$ we include a MATLAB ${ }^{11}$ program used to obtain Figs. 5 and 6; with appropriate modifications the program can be used to produce Figs. 3 and 4 .

We now apply this procedure to calculate and visualize the DOS for particles in rigid boxes with various geometries.

\section{A. Cubical box}

We first will consider a cube of side length $L$.

Table I. The DOS for a particle in a rigid box of $D$ dimensions. The energy $\epsilon$ is in units of the lowest single particle energy $\epsilon_{0}$.

\begin{tabular}{lcc}
\hline \hline$D$ & $\mathcal{N}(\epsilon)$ & $g(\epsilon)=d \mathcal{N} / d \epsilon$ \\
\hline 1 & $\epsilon^{1 / 2}$ & $\frac{1}{2} \epsilon^{-1 / 2}$ \\
2 & $\frac{\pi}{4} \epsilon$ & $\frac{\pi}{4}$ \\
3 & $\frac{\pi}{6} \epsilon^{3 / 2}$ & $\frac{\pi}{4} \epsilon^{1 / 2}$ \\
4 & $\frac{\pi^{2}}{32} \epsilon^{2}$ & $\frac{\pi^{2}}{16} \epsilon$ \\
5 & $\frac{\pi^{2}}{60} \epsilon^{5 / 2}$ & $\frac{\pi^{2}}{24} \epsilon^{3 / 2}$ \\
6 & $\frac{\pi^{3}}{384} \epsilon^{3}$ & $\frac{\pi^{3}}{128} \epsilon^{2}$ \\
\hline \hline
\end{tabular}


Step 1: The energies are $\epsilon=\left(\pi^{2} \hbar^{2}\right)\left(n_{x}^{2}+n_{y}^{2}+n_{z}^{2}\right) /$ $\left(2 M V^{2 / 3}\right)$, where we have used $L^{2}=V^{2 / 3}$ with $V$ the volume of the box. If we set $\left(\pi^{2} \hbar^{2}\right) /(2 M)=1$ and $V=1$, then the energies are all integers.

Step 2: Using Mathematica ${ }^{12}$ we determined the lowest 15,954 energy levels. To calculate the energies we need all the triplets $\left\{n_{x}, n_{y}, n_{z}\right\}$ within the sphere of radius $n_{\max }$ in $n$-space. We make a list of $n_{x}^{2}+n_{y}^{2}+n_{z}^{2}$ with $1 \leq n_{x} \leq n_{\max }$, $1 \leq n_{y} \leq \sqrt{n_{\max }^{2}-n_{x}^{2}}$, and $1 \leq n_{z} \leq \sqrt{n_{\max }^{2}-n_{x}^{2}-n_{y}^{2}}$; this list has all energies with $\epsilon \leq n_{\max }^{2}$ (we chose $n_{\max }=32$ ). This is a very degenerate system-of the 15,954 energy levels only 818 are distinct and the average degeneracy is 19.50 . As seen in Fig. 6, for $\epsilon \sim 1000$ we have $d_{\epsilon} \sim 60$. Specifically, the energies from 933-954, along with their degeneracies, are given by: $\{\epsilon, g(\epsilon)\}=\{933,24\},\{934,39\},\{936,24\}$, $\{937,27\},\{938,24\},\{\},\{940,6\},\{941,66\},\{942,18\}$, $\{944,9\},\{945,48\},\{946,24\},\{947,15\},\{948,18\},\{949$, $12\},\{950,63\},\{952,12\},\{953,45\},\{954,42\}$.

Step 3: Plot $\mathcal{N}(\epsilon)$. It is instructive to construct the set of points for the plot explicitly using the list of energies. There are only 818 steps, one for each value of $\epsilon$. The height of the step at some energy $\epsilon$ is $d_{\epsilon}$, the degeneracy, so this staircase has steps that get higher with energy (e.g., the step at $\epsilon=950$ has a height of 63).

Step 4: While calculating the DOS, the window size needs to be adjusted to give a reasonable-looking graph. If the window is too small, the graph will look noisy; if it is too big, the graph may appear too coarse. We use trial and error to select an appropriate window size. We have included code in the online supplement ${ }^{10}$ in which the reader can select the maximum energy and window size and generate plots like Figs. 5 and 6.

Figures 5 and 6 display $d(\epsilon), \mathcal{N}(\epsilon)$, and $g(\epsilon)$ for this system. Again, it is evident that the agreement between the analytic result and the explicit counting of energies improves as the energy interval increases.

\section{B. Rectangular box}

The rigid rectangular box is a straightforward adjustment to the cubical case and gives us a look at a non-degenerate spectrum. In order to remove the degeneracies from the single-particle spectrum that exist in the cubical case, we will make the sides of the box have incommensurate lengths. The choice $L_{x} \neq L_{y} \neq L_{z}$ with $L_{x}=1, L_{y}=2 / e$, and $L_{z}=e / 2$ gives us a box of unit volume and a non-degenerate spectrum.

Step 1: The energies are $\epsilon=\left(\pi^{2} \hbar^{2}\right)\left[\left(n_{x} / L_{x}\right)^{2}+\left(n_{y} / L_{y}\right)^{2}\right.$ $\left.+\left(n_{z} / L_{z}\right)^{2}\right] /(2 M)$. Again, the obvious choice for our energy scale is to set $\pi^{2} \hbar^{2} /(2 M)=1$.

Step 2: This time we use a different method than in the cubical case. The sphere in $n$-space corresponds to an ellipse in phase space. This is a nice illustration of the difference between these abstract spaces. The method used was an exhaustive calculation of all energies corresponding to the points $1 \leq n_{x} \leq n_{\max }, 1 \leq n_{y} \leq n_{\max }$, and $1 \leq n_{z} \leq n_{\max }$, after which we select $\epsilon \leq\left(2 n_{\max } / e\right)^{2}$. The fact that $n_{y}$ and $n_{z}$ are multiplied by a factor of 0.74 and 1.36 , respectively, ensures that the sphere radius of $\sqrt{2 / e} n_{\max }$ is enclosed in the ellipse in phase space, and we had a complete set of energies. Although the method is inefficient, it has the advantage of transparency.

All the energies in this system are unique. In order to have degeneracies, we would need two sets of integers, $\left\{n_{x}, n_{y}, n_{z}\right\}$ and $\left\{m_{x}, m_{y}, m_{z}\right\}$, such that $n_{x}^{2}+e^{2} n_{y}^{2} / 4+4 n_{z}^{2} / e^{2}=m_{x}^{2}$ $+e^{2} m_{y}^{2} / 4+4 m_{z}^{2} / e^{2}$, which is impossible as $e$ is transcendental. The lowest 62,440 energies of the system are calculated.

Step 3: The cumulative state number $\mathcal{N}(\epsilon)$ consists of steps of unit height, though the widths of the steps (level spacings) vary. For our rectangular box there are 41,435 steps, one for each energy. A plot of $d_{\epsilon} \mathrm{vs} \epsilon$ is a series of horizontal spikes of height 1 .

Step 4: In Fig. 7, we show $g(\epsilon)$ and $\mathcal{N}(\epsilon)$ for this system.

\section{Spherical box}

The energies for a rigid sphere of radius $R$ are $\epsilon_{l n}=\hbar^{2} k_{l n}^{2} /$ $\left(2 M R^{2}\right)$, where $k_{l n}$ is the $n$th zero of $j_{l}(r)$, the $l$ th spherical Bessel function. ${ }^{13}$ Each $\epsilon_{l n}$ has a degeneracy of $2 l+1$. It is easy to confuse $j_{l}(r)$ with $J_{l}(r)$, the ordinary Bessel function, also known as the Bessel function of the first kind; the two are related by ${ }^{13} j_{l}(r)=\sqrt{\pi /(2 r)} J_{l+1 / 2}(r)$. Note that the $n$th zero of $J_{l}(r)$ is written $K_{l n}$. MATHEMATICA has a useful add-on called NumericalMath'BesselZeros' that lists the zeros of the various Bessel functions. In Sec. VI we define the problem of the rigid cylindrical box, where the energies are proportional to $K_{l n}^{2}$, so to avoid confusion we provide the following check: the first three zeros of $J_{0}(r)$ are $\{2.4048$, $5.5201,8.6537\}$, and the first three zeros of $j_{0}(r)$ are $\{3.9374,7.8748,11.8122\}$. Actually, the distinction between $K_{l n}$ and $k_{l n}$ is not important as far as $g(\epsilon)$ goes for the sphere and the cylinder, as the two sets of zeros are interspersed. ${ }^{14}$

Step 1: The role of $\pi$ in the energies of the rectangular wells is taken over by the $k_{l n}$. In the rectangular box, the
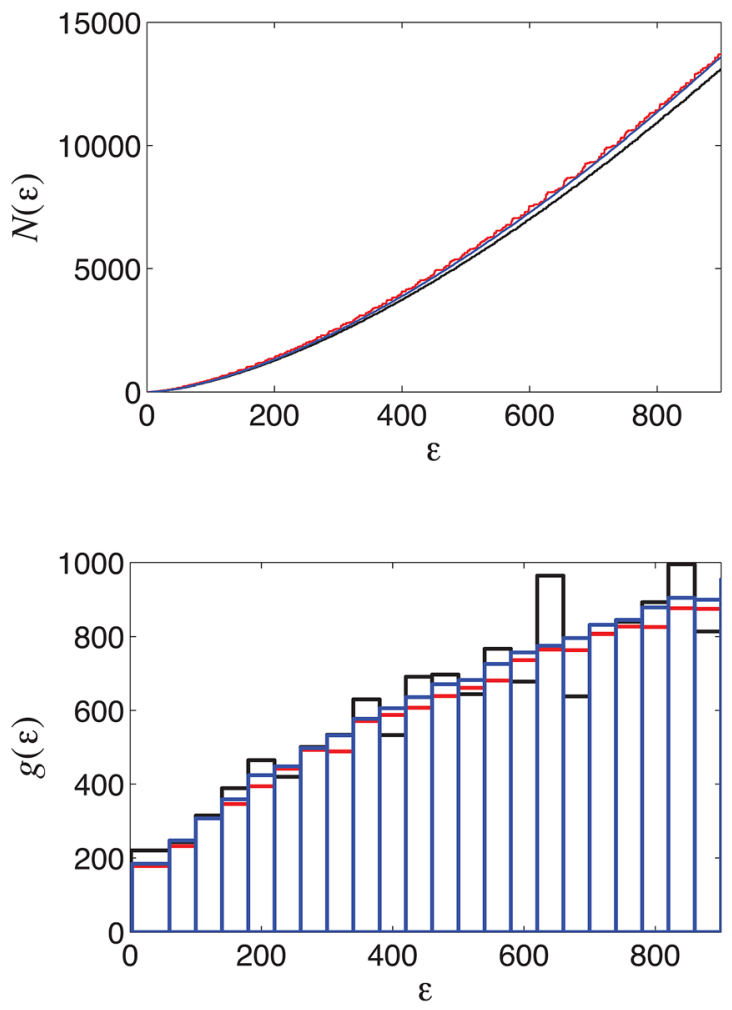

Fig. 7. The upper panel shows the cumulative state number for the spectra for a single particle in cubical, rectangular, and spherical boxes. The spectra have been normalized so that $\hbar^{2} \pi^{2} /(2 M)=1$ and the volume of each box is 1. The three curves are essentially indistinguishable. The lower panel is a histogram of the energies for each box. The bins are 40 units wide. 
zeros of the sine function are multiples of $\pi$. This allowed us to factor it out and choose the energy scale to be in units of $\hbar^{2} \pi^{2} /(2 M)$ for the rectangular boxes. Now, we have $\epsilon=\hbar^{2}\left(k_{l n} / R\right)^{2} /(2 M)$. To compare with the square wells we use an energy scale with $\hbar^{2} \pi^{2} /(2 M)=1$; in these units $\epsilon_{l n}=\left[k_{l n} /(\pi R)\right]^{2}$. Also, if the volume of the box is to be unity, then $R=[3 /(4 \pi)]^{1 / 3}$, so finally we have $\epsilon_{l n}=k_{l n}^{2} /$ $\left(3 \pi^{2} / 4\right)^{2 / 3}$. This is the spectrum we will compare to those of the rectangular and cubical boxes.

Step 2: We calculate all energies with $k_{l n}<95$. To ensure a complete set, we use the brute force method and calculate all the triplets $\left\{k_{l n}, l, n\right\}$ with $0 \leq l \leq 95$ and $1 \leq n \leq 95$, sort these 9,120 numbers, and select the first 95. Sorting triplets as opposed to just the list of $k_{l n}$ makes it easy to accommodate the $2 l+1$ degeneracy when making the final spectrum.

Steps 3 and 4: See Fig. 7.

\section{Weyl's theorem}

It is clear from Sec. II and Fig. 7 that the general trend for the three boxes we examined is $\mathcal{N}(\epsilon)=a \epsilon^{b}$. The energy exponent does not vary much with the shape of the box. A plot of $\ln \mathcal{N}(\epsilon)$ vs $\ln \epsilon$ illustrates this nicely, as shown in Fig. 8. The deviation from the straight line occurs only at low energies, where the shape of the box matters. Using MATHEMATICA, we perform linear fits to the logarithmic plots and obtain the results shown in Table II.

If the box is sufficiently large, a particle in a rigid box should not be aware of the particular shape of the box, so long as $\lambda^{D} \ll V$, where $V$ is the volume of the box. Here, $\lambda=2 \pi /|\vec{k}|$ is the deBroglie wavelength $(|\vec{p}|=\hbar|\vec{k}|)$ and $D$ is the dimension of the space. A slow particle will know about the edge, as it has a long wavelength, and a fast particle or high-energy state will not be sensitive to the shape because its wavelength is small. This is the content of Weyl's theorem, ${ }^{15}$ which can be paraphrased as "high energy eigenvalues of the wave equation are insensitive to the shape of the boundary." A nice account of the origin of the theorem is given by $\mathrm{Kac}^{16}$ and an explicit proof at the level of this paper for the case of the cylinder and sphere is given by Lambert. ${ }^{17}$

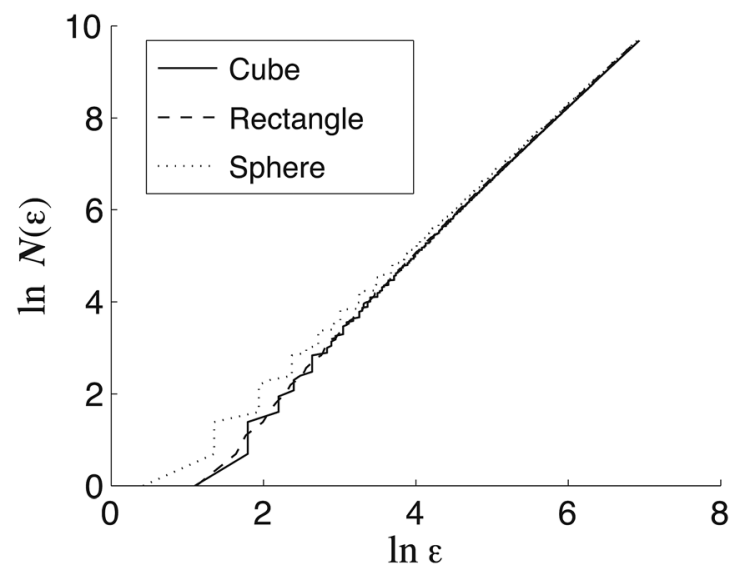

Fig. 8. A logarithmic plot of the cumulative state number for the singleparticle spectra of cubical, rectangular, and spherical boxes. The energies are in units of $\hbar^{2} \pi^{2} /(2 M)$ and the volume of each box is 1 .
Table II. Parameters for $\mathcal{N}(\epsilon)=a \epsilon^{b}$ for the three different 3-D "boxes." The theory gives $a=\pi / 6 \approx 0.524$ and $b=3 / 2$; see Table I.

\begin{tabular}{lcc}
\hline \hline & $a$ & $b$ \\
\hline Cube (theory) & $\pi / 6 \approx 0.524$ & $3 / 2=1.5$ \\
Cube (numerical) & 0.29 & 1.58 \\
Rectangular box & 0.32 & 1.56 \\
Spherical box & 0.46 & 1.51 \\
\hline \hline
\end{tabular}

\section{IV. $N$ PARTICLES: STATISTICAL MECHANICS}

Statistical mechanics can be introduced by analyzing a model system of $N$ non-interacting particles on a singleparticle spectrum, often chosen as a set of equally spaced levels. ${ }^{7}$ This is a valuable pedagogical tool for exploring the statistical distributions for classical particles, identical bosons, and identical fermions. This type of model is rich with subtleties - the particles not only don't see each other beyond obeying the Pauli exclusion principle if they are identical fermions, but their presence has no influence on the energies of the levels they fill. ${ }^{18}$ Real particles, on the other hand, have interactions, so how does this model give useful results? One can think of the effect of all the other particles as being a smooth mean field that can be modeled as a potential for that single particle. Another subtlety is the robustness of the spectra of these $N$-body systems-they are very insensitive to the details of the single-particle spectrum upon which they are built, depending only on its DOS.

We made a simple model of the $N$-particle system to serve as a starting point for students to explore what happens to the DOS as $N$ increases, and to illustrate that the DOS of many-body systems like this are insensitive to the details of the single-particle spectra they are based on. In this section, $E$ is used for the total energy of the system, reserving $\epsilon$ for the single-particle energy. Subscripts will be used on $g$ and $\mathcal{N}$ to indicate the number of particles in the system, so $g_{7}(E)$ is the DOS for a system of seven particles. We compare $N$-particle systems built on four distinct single-particle spectra: the square, rectangular, and spherical 3-D boxes, and a spectrum with a specified DOS but random levels. A simple analytical model for the DOS is presented and compared with the numerical results. The focus is on the relationship between the original single-particle energies that the $N$ particles populate and the resulting $g_{N}(E)$. The model demonstrates that $g_{N}(E)$ depends on the density of the single-particle spectrum, as opposed to the details of the energies themselves; this is why we can replace the exact single-particle energies (zeros of Bessel functions, etc.) with any set of numbers as long as they have the same range and density.

Using a simple algorithm, the spectrum of $N$ identical bosons on a set of single-particle energies $\left\{\epsilon_{i}\right\}$ is calculated. The range of energies is $0 \rightarrow E_{\max }$, with $E_{\max }$ limited only by computational power and user patience. The choice of bosons avoids the subtleties of Fermi statistics. The algorithm is best illustrated with an example. Let's look at identical bosons on the single-particle energies of the rigid cube in three dimensions. First we make a list of the single-particle-state energies: $\left\{\epsilon_{i}\right\}=\{3,6,6,6,9,9,9,11,11,11,12,14$, $14, \ldots\}$. We choose $E_{\max }=53$ so the list includes the first 140 states. This means that we are guaranteed to have a complete list of energies up to $E=53$. For our purposes an $N$-particle state is a list of occupied single-particle states, so 
$(4,5,5,27)$ is a four-particle state with a particle in state 4, two particles in state 5 , and a particle in state 27 . Now list the indices of all possible one-particle states: $\{(i)\}=\{(1)$, $(2),(3), \ldots,(140)\}$; the energy of state $(i)$ is $\epsilon_{i}$. Next list the index pairs of all possible two-particle states: $\{(i, j)\}=\{(1,1),(1,2),(1,3), \ldots,(140,140)\}$, sort them and drop duplicates; the energy of the state $(i, j)$ is $\epsilon_{i}+\epsilon_{j}$. Drop all states with $E>53$ as you are only guaranteed a complete set of energies for $E \leq 53$. In general, list all possible $N$-particle states, sort them, drop duplicates, and sum the corresponding single-particle energies. Once you have these spectra you can use the procedure in Sec. III to get $g_{N}(E)$ and $\mathcal{N}_{N}(E)$.

Systems based on a random single-particle spectrum are included for comparison with those based on the rigid boxes because this removes the role of geometry. The distribution of the random numbers is such that the DOS is $g(\epsilon)$ $=(\pi / 4) \epsilon^{1 / 2}$; this is accomplished by taking a set $\left\{x_{i}\right\}$ of 500 random numbers uniformly distributed on the interval $[0,1]$, sorting them, raising them to the power of $2 / 3$, and multiplying them by $[(6 / \pi) 500]^{2 / 3}$. The logic behind this is worth mentioning. Given a set of numbers $\{x\}$ with distribution $f(x)$, we can make a function $y(x)$ such that the set $\{y(x)\}$ has a distribution $\alpha y^{\beta}$ by noting that $f(x) d x=\alpha y^{\beta} d y$. In our case $f(x)=1$, and we have $d y / d x=(1 / \alpha) y^{-\beta}$ so that $y(x)=[(\beta+1) / \alpha]^{1 /(\beta+1)} x^{1 /(\beta+1)}$.

All of these systems have a cumulative state number of the form $\mathcal{N}_{N}(E)=\alpha E^{\beta}$, with both $\beta$ and $\ln \alpha$ being linear in $N$. In Fig. 9, we show $\mathcal{N}_{N}(E)$ vs $E$ for $N$ particles on the single-particle energies of a spherical well, while Figs. 10 and 11 show graphs of $\beta$ and $\ln \alpha$ as functions of $N$ for the various systems we examined. The values of $\alpha$ and $\beta$ for the random spectra are based on 100 separate single-particle spectra.

Given a single-particle spectrum with a one-particle DOS $g_{1}(E)$, we can obtain a naive expression for the $N$-body density of states $g_{N}(E)$ iteratively. The density of $N$-body states with energy $E$ is a product of the density of $(N-1)$-body states with energy $E^{\prime}$ and one-body states with energy $E-E^{\prime}$, or

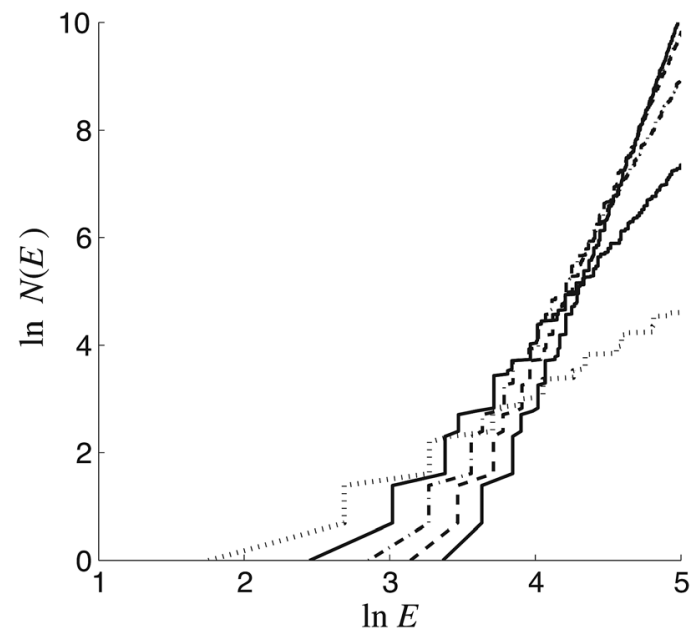

Fig. 9. A logarithmic plot of the cumulative state number for $N$-particle systems $(N=1,2, \ldots, 5)$ on the single-particle spectrum of the sphere. The steeper curves correspond to higher values of $N$, and different line types have been used for clarity. The slopes follow $N(b+1)$ [see Eq. (9)].

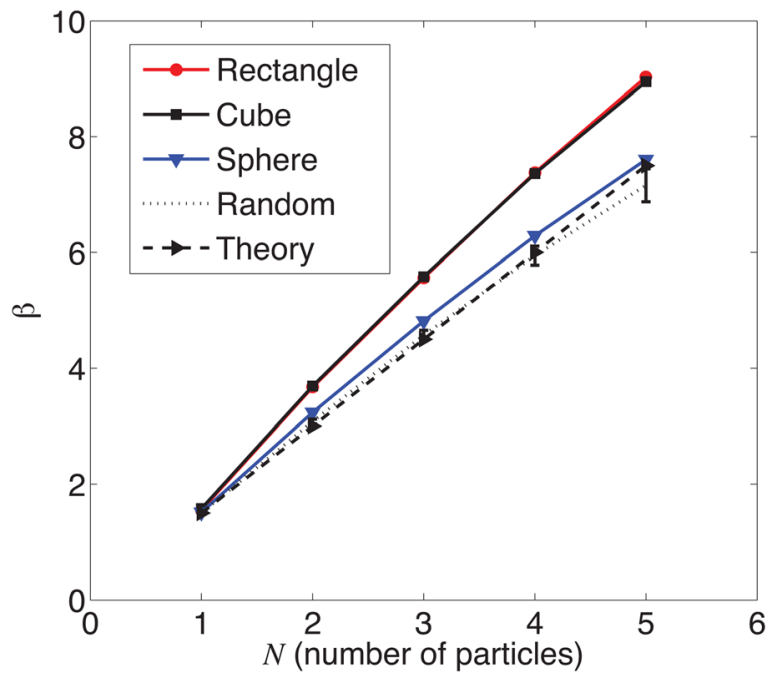

Fig. 10. The exponent of $E$ in $\mathcal{N}(E)=\alpha E^{\beta}$ for systems of various particle number. Each system is constructed by placing non-interacting particles on the corresponding single-particle spectrum described in the text. The dashed line is a theoretical value derived using the values $a=0.4$ and $b=1 / 2$ in Eq. (8). These results are obtained using a linear fit to the $\ln \mathcal{N}(E)$ data. The error bars represent the standard deviation of the average values from the 100 random spectra. (The lines are a guide for the eye.)

$$
g_{N}(E)=\int_{0}^{E} g_{N-1}\left(E^{\prime}\right) g_{1}\left(E-E^{\prime}\right) d E^{\prime} .
$$

This expression double-counts some states. Suppose we add to the three-particle state $\left\{n_{1}, n_{2}, n_{3}\right\}=\{3,17,17\}$ a fourth particle in state 24 ; then $E=\epsilon_{3}+2 \epsilon_{17}+\epsilon_{24}$, and there is a contribution to the integral in Eq. (6) from $g_{1}\left(\epsilon_{24}\right) g_{3}\left(\epsilon_{3}+2 \epsilon_{17}\right)$. However, we can get to the same state by adding a particle in state 17 to the three-particle state $\left\{n_{1}\right.$, $\left.n_{2}, n_{3}\right\}=\{3,17,24\}$, and this gives a contribution to the integral in Eq. (6) from $g_{1}\left(\epsilon_{17}\right) g_{3}\left(\epsilon_{3}+\epsilon_{17}+\epsilon_{24}\right)$. This is the origin of the double counting and its effect is to increase the number of states by a number close to $N$ at high energies, where the occupancies of the single-particle states are low. We did not fix this problem.

When iterating Eq. (6) we need the following result for integer $n$ and $m$ :

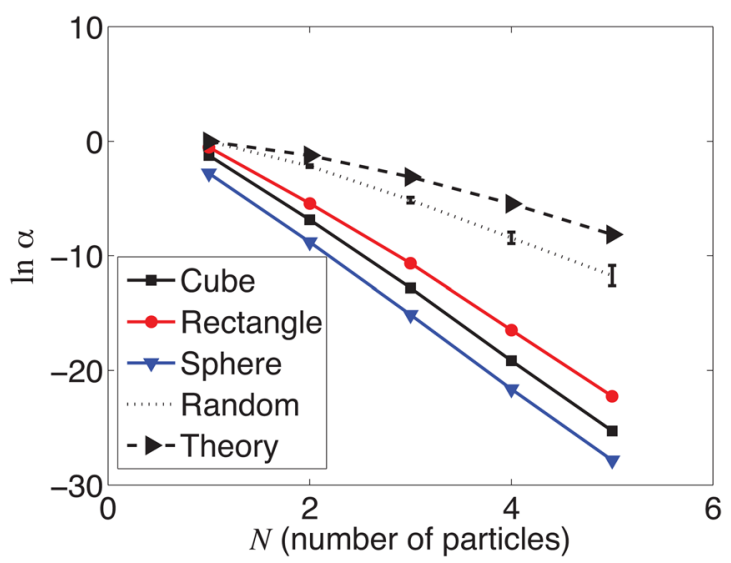

Fig. 11. A graph of $\ln \alpha$ vs $N$ for the same systems as in Fig. 10. Again, these results are obtained using a linear fit to the $\ln \mathcal{N}(E)$ data. The error bars represent the standard deviations of the average values from the 100 random spectra. (The lines are a guide for the eye.) 
Table III. The DOS for $N$-particle systems built on single-particle spectra with a DOS $g_{1}(E)=a E^{b}$. We set $a=1$ for simplicity but $g_{N}(E)$ includes a factor of $a^{N}$.

\begin{tabular}{lcccc}
\hline \hline & $b=-1 / 2$ & $b=0$ & $b=1 / 2$ & $b=1$ \\
\hline$g_{1}(E)$ & $E^{-1 / 2}$ & 1 & $E^{1 / 2}$ & $E$ \\
$g_{2}(E)$ & $\pi$ & $E$ & $\frac{\pi}{8} E^{2}$ & $\frac{1}{3 !} E^{3}$ \\
$g_{3}(E)$ & $2 \pi E^{1 / 2}$ & $\frac{1}{2 !} E^{2}$ & $\frac{2 \pi}{105} E^{7 / 2}$ & $\frac{1}{5 !} E^{5}$ \\
$g_{4}(E)$ & $\pi^{2} E$ & $\frac{1}{3 !} E^{3}$ & $\frac{\pi^{2}}{1920} E^{5}$ & $\frac{1}{7 !} E^{7}$ \\
$g_{5}(E)$ & $\frac{4 \pi^{2}}{3} E^{3 / 2}$ & $\frac{1}{4 !} E^{4}$ & $\frac{4 \pi^{2}}{135135} E^{13 / 2}$ & $\frac{1}{9 !} E^{9}$ \\
$g_{6}(E)$ & $\frac{\pi^{3}}{2} E^{2}$ & $\frac{1}{5 !} E^{5}$ & $\frac{\pi^{3}}{2580480} E^{8}$ & $\frac{1}{11 !} E^{11}$ \\
$g_{7}(E)$ & $\frac{8 \pi^{3}}{15} E^{5 / 2}$ & $\frac{1}{6 !} E^{6}$ & $\frac{8 \pi^{3}}{654729075} E^{19 / 2}$ & $\frac{1}{13 !} E^{13}$ \\
$g_{8}(E)$ & $\frac{\pi^{4}}{6} E^{3}$ & $\frac{1}{7 !} E^{7}$ & $\frac{\pi^{4}}{10218700800} E^{11}$ & $\frac{1}{15 !} E^{15}$ \\
$\vdots$ & & & $\frac{\pi^{N / 2}}{2^{N}(3 N / 2-1) !} E^{3 N / 2-1}$ & $\frac{1}{(2 N-1) !} E^{2 N-1}$ \\
$g_{N}(E)$ & $\frac{\pi^{N / 2}}{(N / 2-1) !} E^{N / 2-1}$ & $\frac{1}{(N-1) !} E^{N-1}$ &
\end{tabular}

$$
\int_{0}^{E}\left(E^{\prime}\right)^{n}\left(E-E^{\prime}\right)^{m} d E^{\prime}=\frac{n ! m !}{(n+m+1) !} E^{n+m+1} .
$$

Table III gives several examples of the DOS for $N$ bosons based on single-particle spectra with level density $g_{1}(E)=E^{b}$, for $b=-1 / 2,0,1 / 2$, and 1 , corresponding to a box in 1, 2, 3, and 4 dimensions [see Eq. (5)]. In general, after iterating Eq. (6) for $N$ bosons on a single-particle spectrum with $g_{1}(E)=a E^{b}$, we get

$$
\begin{aligned}
& g_{N}(E)=\frac{b !^{N}}{(N b+N-1) !} a^{N} E^{N b+N-1}, \\
& \mathcal{N}_{N}(E)=\frac{b !^{N}}{(N b+N) !} a^{N} E^{N b+N}=\alpha E^{\beta} .
\end{aligned}
$$

This simple expression matches the numerical results for the behavior of $\alpha$ and $\beta$. In Figs. 9 and 10, we see that $\beta$ is linear in $N$. The systems built on the single particle levels of both the sphere and random system are close to the theoretical expression with $a=0.4$ and $b=0.5$ (we chose those values based on Table II). In Fig. 11, we see that $\ln (\alpha)$ is approximately linear in $N$; however, we cannot account for the relative sizes of $\ln (\alpha)$. Intuitively one would expect a smaller $a$ to give a smaller $\alpha$, so the values in Table II would suggest that the sphere systems would be higher than the cube systems in Fig. 11, which is not the case.

\section{CONCLUSIONS}

We have provided a graphical approach to introduce students to the density of states (DOS) for simple quantum systems. In Sec. I, we introduced the concepts of degeneracy, cumulative state number $\mathcal{N}(\epsilon)$, and DOS $g(\epsilon)$ for these systems. In Sec. III, we gave a procedure for visualizing the DOS and used it to get the DOS for a particle in rigid boxes of various shapes. We found that the shape of the box had little effect on the DOS, a demonstration of Weyl's theorem. We then built $N$-particle systems on the single-particle spectra of Sec. III and found that the DOS was insensitive to the specific energies of the single-particle spectra, the singleparticle DOS $\left(g_{1}(\epsilon)\right)$ being the important factor. Finally, we compared our numerical results for $\mathcal{N}_{N}(E)$ with an expression based on iterating the single-particle density of states and saw how the DOS for $N$-particle systems was simply related to $g_{1}(\epsilon)$.

\section{ADDITIONAL PROBLEMS}

The following problems provide additional practice with the procedure used in Sec. III. The programs available in Ref. 10 may be a helpful starting point.

1. Examine changes to the density of states if we continuously change one of the dimensions of the rigid rectangular box. We expect the density of states to exhibit different behavior as we change continuously from a "2D" system $\left(L_{z} \ll L_{x}, L_{y}\right)$, to a "3-D" system $\left(L_{z} \approx L_{x}, L_{y}\right)$ to a "1-D" system $\left(L_{z} \gg L_{x}, L_{y}\right)$. Let $L_{x}=L_{y}=L_{0}=1$, and then let $L_{z}$ vary over the range $0.01 L_{0} \leq L_{z} \leq 100 L_{0}$.

2. The energy levels for a particle in a rigid cylindrical box of height $H$ and radius $R$ are given by ${ }^{13} \epsilon_{q l n}=\hbar^{2}$ $\left(q^{2} \pi^{2} / H^{2}+K_{l n}^{2} / R^{2}\right) /(2 M)$, where the $K_{l n}$ are zeroes of the (regular) Bessel functions. Vary the height-to-radius ratio for the cylindrical box. As $H / R$ goes from near 0 to 1 , you should expect a transition from "2-D" behavior to "3-D" behavior. What does this look like in terms of $g(\epsilon)$ ?

3. Redo the treatment of Sec. II for a relativistic particle in the 2-D rigid square box. The important difference is that relativistically we have $\epsilon=p c=\hbar k c$, where $k$ is the wave number and $c$ is the speed of light.

\section{ACKNOWLEDGMENTS}

The authors thank T. Bensky for helpful discussions. For their hospitality during sabbatical leaves M.J.M. thanks the faculty of the School of Physics at the Dublin Institute of Technology and D.M. is grateful to the physics department at Temple University. The reviewers and editors provided detailed comments that significantly clarified our presentation.

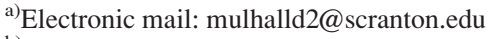

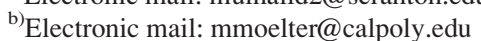

${ }^{1}$ Daniel Schroeder, An Introduction to Thermal Physics (Addison-Wesley, San Francisco, 2000).

${ }^{2}$ Ralph Baierlein, Thermal Physics (Cambridge, New York, 1999).

${ }^{3}$ P. C. Riedi, Thermal Physics, 2nd ed. (Oxford, New York, 1988).

${ }^{4}$ Frederick Reif, Fundamentals of Statistical and Thermal Physics (McGraw-Hill, New York, 1965).

${ }^{5}$ Franz Mandl, Statistical Physics, 2nd ed. (Wiley, New York, 1988).

${ }^{6}$ Charles Kittel and Herbert Kroemer, Thermal Physics, 2nd ed. (Freeman, New York, 1980).

${ }^{7}$ Raymond A. Serway, Clement J. Moses, and Curt A. Moyer, Modern Physics, 3rd ed. (Thompson Brooks/Cole, 2005).

${ }^{8}$ This visual approach is discussed in Ref. 2 on pp. 75-79. A related treatment from the momentum viewpoint is in Appendix IV of Ref. 3, pp. 297-304.

${ }^{9}$ Claude Garrod, Statistical Mechanics and Thermodynamics (Oxford, New York, 1995).

${ }^{10}$ See supplementary material at http://dx.doi.org/10.1119/1.4867489 for sample programs demonstrating how our calculations are carried out are available.

${ }^{11}$ MatLaB software from The Mathworks, <www.mathworks.com>.

${ }^{12}$ Mathematica software from Wolfram Research, <www.wolfram.com $>$.

${ }^{13}$ Richard L. Liboff, Introductory Quantum Mechanics (Holden-Day, 1980).

${ }^{14}$ Richard L. Liboff, "Density of states and other quantum properties of a spherical cavity," Phys. Rev. A 43(11), 5765-5769 (1991).

${ }^{15} \mathrm{H}$. Weyl, "Das asymptotische Verteilungsgesetz der Eigenwerte linearer partieller Differentialgleichungen," Math. Ann. 71, 441-479 (1912).
} 
${ }^{16}$ Mark Kac, "Can one hear the shape of a drum?," Am. Math. Mon. 73(4), 1-23 (1966).

${ }^{17}$ R. H. Lambert, "Density of states in a sphere and cylinder," Am. J. Phys. 36(5), 417-420 (1968).

${ }^{18}$ Anecdotally, one of the authors (D. M.) has been surprised by the persistent student misconception that "fermions don't interact." This false notion can be traced to the commonly used "system of noninteracting fermions" that introduces statistical mechanics in the intermediate course. The equidistant single-particle spectrum is a common starting point when looking at the spectra of many-body systems. It is confusing that after all the work invested in solving the single-particle Schrödinger equation, we just throw away our hard won zeros-of-Bessel-functions-based energies, and replace them with the integers.

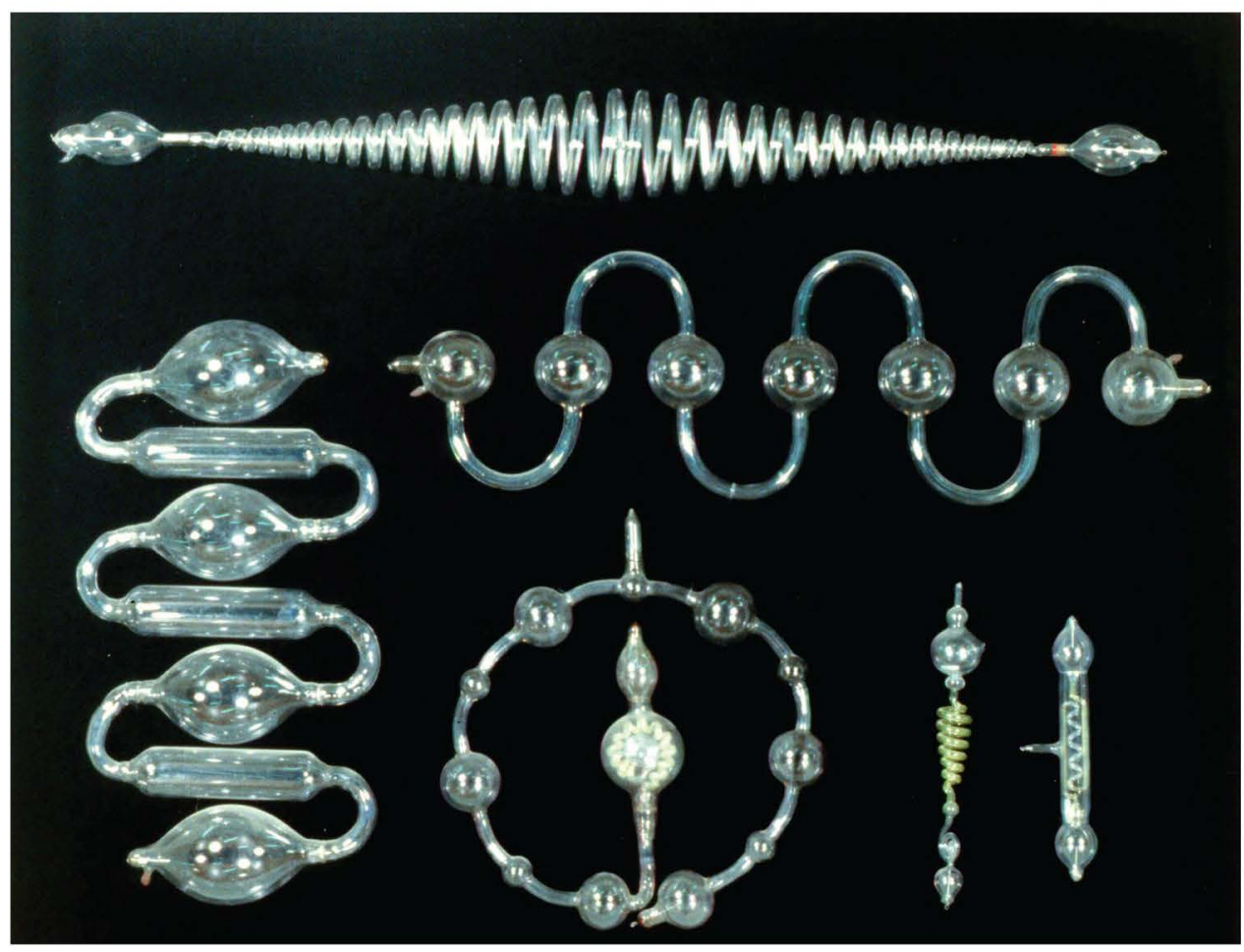

Geissler Tube Set

"Geissler Tube" is a generic name for any discharge tube: an evacuated glass enclosure with two or more metallic electrodes passing through the walls. Some of the tubes are utilitarian, and some are just for enjoyment. Johann Heinrich Wilhelm Geissler (1815-1879) came from a glass-blowing family. About 1852 he became a maker of scientific instruments in Bonn, and in 1855 he constructed a vacuum pump that used droplets of mercury falling through a tube as a method of producing relatively high vacua. This pump was used to evacuate the discharge tubes that he made a few years later for Julius Plücker. These tubes contained rarified gases, and the discharge was obtained in a narrow channel between the electrodes at either end of a straight tube. We still use discharge tubes in undergraduate laboratory work on spectra of gases. This set of fanciful Geissler tubes was shown to me at Dartmouth College in 1979 by Prof. Allen King, who was responsible for the preservation of the historical physics apparatus at his institution. (Notes and photograph by Thomas B. Greenslade, Jr., Kenyon College) 